\title{
Interfacial effects on nucleate boiling heat transfer of binary mixtures
}

\author{
Li He Chai ${ }^{a}$, Xiao Feng Peng ${ }^{a *}$, D.J. Lee ${ }^{b}$ \\ a Thermal Engineering Department, Tsinghua University, Beijing 100084, China \\ b Chemical Engineering Department, Taiwan University, Taipei, Taiwan 106
}

(Received 12 January 2000, accepted 16 March 2000)

\begin{abstract}
Experimental and theoretical analyses were conducted to investigate the effects of interfacial behavior on the nucleate boiling heat transfer of ethanol-water, methanol-water, methanol-n-pentane, ethanol-n-pentane and methanol-ethanol binary mixtures. It is demonstrated that binary mixture vapor-liquid interfacial behavior greatly influences boiling dynamics, such as bubble detachment, flow or heat transfer in microlayer, and stability of microlayer. The associated nucleate boiling heat transfer of binary mixtures will, thus, be significantly altered by Marangoni effect caused by surface tension gradient. The experimental results would not correlate with theoretical results if the Marangoni effects at interface had not been considered. (C) 2001 Éditions scientifiques et médicales Elsevier SAS
\end{abstract}

interface / binary mixture / boiling / heat transfer / Marangoni / bubble dynamics

\section{Nomenclature}

$A$

$b$

c

C

C

$g$

$h$

H

L latent heat . . . . . . . . . . .

$m$ mass flow rate ............

Ma Marangoni number

$\mathrm{Nu}$ Nusselt number

$P \quad$ pressure ...............

Pr Prandtl number

$r$ radial coordinate ...........

$r_{0}$ equilibrium bubble radius . . . . . . .

$q$ heat flux ..............

$T$ temperature ............

\footnotetext{
* Correspondence and reprints.

E-mail address: pxf-dte@ $@$ tsinghua.edu.cn (X.F. Peng).
}

voltage ............. $\quad \mathrm{V}$

$x \quad$ mass percentage

$x \quad$ coordinate ............ m

$y$ mass percentage

$z$ coordinate ............ m

Greek symbols

$\Delta T$ liquid superheat $\ldots \ldots \ldots$

$\mathrm{m} \cdot \mathrm{s}^{-2} \quad \alpha \quad$ wave number

$\mathrm{W} \cdot \mathrm{m}^{-2} \cdot \mathrm{K}^{-1} \quad \beta \quad$ wave growth rate

$\mathrm{m}$

A

$\mathrm{J} \cdot \mathrm{kg}^{-1}$

$\mathrm{kg} \cdot \mathrm{s}^{-1}$

$\mathrm{MPa}$

surface tension ...........

$\mathrm{N} \cdot \mathrm{m}^{-1}$

dimensionless variable

density

$\mathrm{kg} \cdot \mathrm{m}^{-3}$

dimensionless variable

thermal conductivity . . . . . . . W W $\mathrm{m}^{-1} \cdot \mathrm{K}^{-1}$

$\gamma \quad$ activity coefficient

$\mu$ viscosity

$\mathrm{kg} \cdot \mathrm{m}^{-1} \cdot \mathrm{s}^{-1}$

\section{Subscripts}

$\begin{array}{lll}\mathrm{m} & & \text { liquid } \\ \mathrm{m} & 1\end{array}$

$\mathrm{W} \cdot \mathrm{m}^{-2}$ e excess

$\mathrm{K} \quad \mathrm{i}$ interface, ideal state

$i \quad i=1,2$

j $j=1,2$

$\mathrm{p}$ constant pressure

$\mathrm{s}$ saturation 
sf solid-liquid

v vapor

w wall surface

1 component 1

2 component 2

\section{INTRODUCTION}

Binary mixtures boiling is of particular importance in the chemical and process industries, and recently binary mixtures are also used as working fluids in thermal power plants and refrigeration systems due to their potential to reduce available energy loss in heat exchangers. Efforts to improve component or system performance have led researchers to consider the binary working mixtures. They have found that some binary mixtures offer potential for improved thermodynamic efficiency and superior heat transfer performance. The significance of binary mixtures is more evident in the thermal management systems for spacecraft, for it may be possible to enhance the ability of the system to resist dry-out and the onset of film boiling [1].

The importance of boiling in a wide variety of applications has provided the incentive for numerous fundamental investigations over the past two decades. There is now a large body of published information regarding experiments on pool boiling of mixtures, and considerable effort has been made to develop physical models describing the phenomena [2]. However, there is still a lack of techniques and methods for the prediction of nucleate pool boiling heat transfer of binary mixtures. Because of the complexity of the subject and lack of knowledge on binary mixture pool boiling, currently available models in the literature are not able to predict the heat transfer with satisfaction. It can be seen that most of these correlations were derived assuming that vapor bubbles grew in a uniformly superheated infinite liquid. However, such an assumption is far from reality for vapor bubbles growing on a heated surface.

It is well known that surface tension is a strong function of component concentration. In the so-called positive binary systems, the volatile component has a lower surface tension. Marangoni phenomena resulting from interface tension gradients will be focused in the present study. Examining the role of Marangoni effect on boiling will not only be beneficial for proposing more satisfactory heat transfer correlations, but will also provide some new insight into our fundamental understanding of the heat transfer mechanism of binary mixtures both under microgravity and under terrestrial conditions.

In this paper, experimental and theoretical analyses were conducted to investigate the effects of interfacial behavior on the nucleate boiling heat transfer of ethanolwater, methanol-water, methanol-n-pentane, ethanoln-pentane and methanol-ethanol binary mixtures. It was found that Marangoni effect plays an important role in nucleate boiling dynamics and associated heat transfer of binary mixtures besides mass transfer resistance. The experimental results would not correlate with theoretical results if the Marangoni effects at interface had not been considered.

\section{THEORETICAL ANALYSIS}

\subsection{Interfacial behavior}

For a binary mixture such as water-ethanol or watermethanol, the surface tension can be adequately expressed as

$$
\sigma=x_{1} \sigma_{1}\left(T_{\mathrm{i}}\right)+\left(1-x_{1}\right) \sigma_{2}\left(T_{\mathrm{i}}\right)
$$

$x_{1}$ is the concentration of component 1 at the interface, which is different from those based on bulk liquid composition. In this case, both growing bubble and its interface are in the concentration boundary layer.

As is the case for most pure liquids, the surface tension decreases almost linearly with temperature. Consequently, curve fit equations for surface tension data are almost linear and the surface tension of the each component are assumed to be linear relation given by Jaspar [3].

The growth of a bubble controlled by heat transfer would stand for the case that bubble grew large enough, so as the vapor pressure in bubbles is nearly equal to saturated pressure of the liquid that may be assumed to be constant. In addition, tangential vapor flow along the bubble interface often occurs rapidly. In terms of this opinion, the vapor pressure of a binary mixture can be expressed as

$$
P_{\mathrm{v}}=x_{1} \gamma_{1} P_{1}^{\mathrm{s}}+\left(1-x_{1}\right) \gamma_{2} P_{2}^{\mathrm{s}}
$$

Here the activity coefficient $\gamma_{i}$ is not unity for most liquid mixtures with strong molecular interactions, which can be usually determined from the two-parameter Wilson equations [4], 


$$
\begin{aligned}
\ln \gamma_{i}= & -\ln \left(x_{i}+x_{j} \lambda_{i j}\right) \\
& +x_{j}\left[\frac{\lambda_{i j}}{x_{i}+x_{j} \lambda_{i j}}-\frac{\lambda_{j i}}{x_{i}+x_{j} \lambda_{j i}}\right]
\end{aligned}
$$

The constants $\lambda_{i j}$ and $\lambda_{j i}$ can be determined from experimental data for mixtures and are independent of temperature over the range of interest.

The vapor pressures of pure components $P_{i}$ are calculated from the Antoine equation [4], i.e.

$$
\ln P_{i}^{\mathrm{s}}=A_{i}-\frac{B_{i}}{T+C_{i}}
$$

where the constants $A_{i}, B_{i}$, and $C_{i}$ can be found in the literature.

Using the chain rule, the following equation is obtained from equation (1):

$$
\begin{aligned}
\frac{\mathrm{d} \sigma}{\mathrm{d} T}= & {\left[\sigma_{1}\left(T_{\mathrm{i}}\right)-\sigma_{2}\left(T_{\mathrm{i}}\right)\right] \frac{\mathrm{d} x_{1}}{\mathrm{~d} T} } \\
& +\left[x_{1} \frac{\mathrm{d} \sigma_{1}}{\mathrm{~d} T}+\left(1-x_{1}\right) \frac{\mathrm{d} \sigma_{2}}{\mathrm{~d} T}\right]
\end{aligned}
$$

Differentiating equation (2) with respect to interfacial temperature $T_{\mathrm{i}}$ and setting the result equal to zero, we have

$$
\begin{aligned}
\frac{\mathrm{d} x_{1}}{\mathrm{~d} T}= & {\left[x_{1} \frac{\mathrm{d} \ln P_{1}^{\mathrm{s}}}{\mathrm{d} T}+\left(1-x_{1}\right) \frac{\gamma_{2}}{\gamma_{1}} \frac{P_{2}^{\mathrm{s}}}{P_{1}^{\mathrm{s}}}\right] } \\
& \cdot\left[\frac{\gamma_{2}}{\gamma_{1}} \frac{P_{2}^{\mathrm{s}}}{P_{1}^{\mathrm{s}}}-1-x_{1} \frac{\mathrm{d} \ln \gamma_{1}}{\mathrm{~d} x_{1}}\right. \\
& \left.-\left(1-x_{1}\right) \frac{\gamma_{2}}{\gamma_{1}} \frac{\mathrm{d} \ln \gamma_{2}}{\mathrm{~d} x_{1}} \frac{P_{2}^{\mathrm{s}}}{P_{1}^{\mathrm{s}}}\right]^{-1}
\end{aligned}
$$

The relation of surface tension varying with temperature can be obtained by substituting equation (6) into equation (5). Marangoni number introduced to describe the interfacial characteristics is in the form as

$$
M a=\frac{-(\mathrm{d} \sigma / \mathrm{d} T)\left(T_{\mathrm{S}}-T_{\mathrm{w}}\right) H}{a \mu}
$$

where $H$ length scale is approximately equal to boundary layer thickness. The results calculated by equation (7) for methanol-water, methanol-n-pentane, ethanol-n-pentane and methanol-ethanol binary mixtures are illustrated in figure 1 . Here $T_{\mathrm{s}}-T_{\mathrm{w}}=-15 \mathrm{~K}$, and $H=5 \mathrm{~mm}$. Very clearly, different binary mixtures display different interfacial behaviors. For positive binary mixtures, such as methanol-water, ethanol-n-pentane before azeotropic and methanol-n-pentane after azeotropic, $M a$ number is

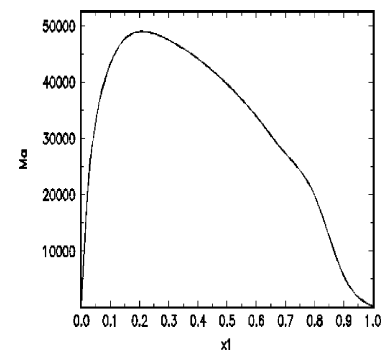

(a)

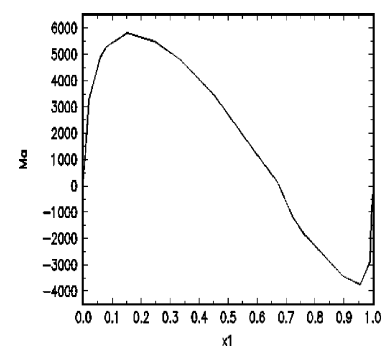

(c)

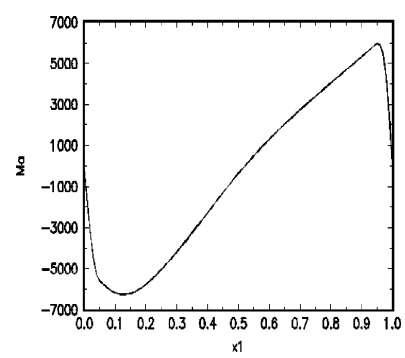

(b)

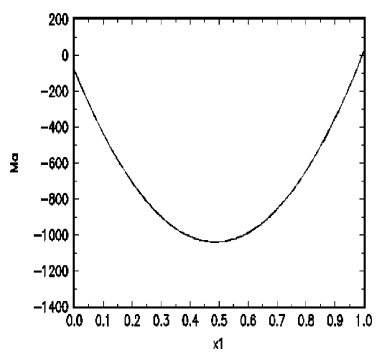

(d)
Figure 1. The variations of $M a$ with compositions $x_{1}$ : (a) watermethanol, (b) n-pentane-methanol, (c) n-pentane-ethanol, and (d) ethanol-methanol.

positive. For negative binary mixtures, such as methanolethanol, ethanol-n-pentane after azeotropic and methanol-n-pentane before azeotropic, $M a$ number is negative. For the surface tension of water is much larger than methanol, $M a$ number for methanol-water mixtures is very large and, thus, has unusual interfacial behaviors. Two extreme values exist for methanol-n-pentane and ethanol-n-pentane binary mixtures because of their azeotropic performance. For methanol-ethanol binary mixture, since the surface tension difference between two mixed compositions is not evident, $M a$ number is small, and thus, Marangoni effect is not significant.

\subsection{Interfacial effect on bubble detachment}

In [5], a microwedge model was proposed for pool nucleate boiling of binary mixtures under microgravity. The microwedge layer interface characteristics were surfaced by solving bubble interface equation. The result is shown in figure 2 . Here $\xi=z / r_{0}, \lambda=r / r_{0}$. Constant $A$ indicates the variations of surface tension along the interface of microwedge layer. $A<0$ denotes positive binary mixture, while $A>0$ denotes negative binary mixture or pure liquid. The characteristics of the microwedge layer interface have a direct relationship with changing of con- 


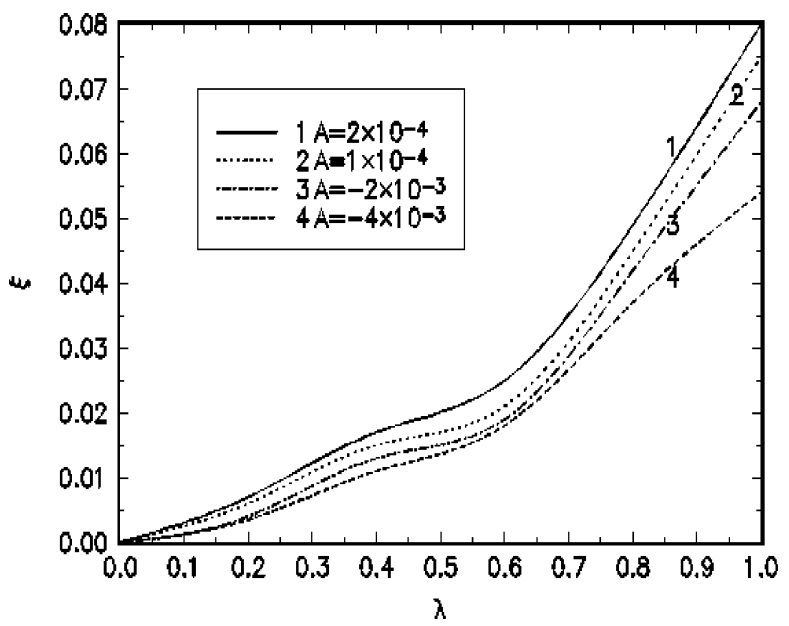

Figure 2. The shape of microlayer interface for different values of $A$.

tact line in a macroscopic sense. As shown in figure 2, for positive binary mixtures, the microwedge layer interface flattens with increasing absolute value of $A$. Variation of surface tension along microwedge layer interface actually results in contact line moving towards the bubble center point at the heated surface, which means that the microwedge layer merges each other underneath the bubble and consequently detaches fully-grown bubble from the heated surface. This might dominate nucleate boiling under microgravity.

\subsection{Interfacial effect on flow and heat transfer in microlayer}

Consider the evaporating microwedge liquid layer, as observed in nucleate boiling of binary mixtures at low heat flux. The liquid in microwedge layer is driven by the interfacial shear resulted from surface tension gradient and capillary force gradient resulted from curvature gradient [6]. The result for methanol-water is shown in figure 3. The results demonstrate that for positive binary mixtures, the interfacial shear caused by surface tension gradient will greatly enhance mass flow rate in microlayer and the associated evaporative heat transfer.

\subsection{Interfacial effect on stability of microlayer}

Perturbation analysis of simplified Navier-Stokes equations for microlayer underneath growing bubble was conducted to investigate the stability of evaporating layer.

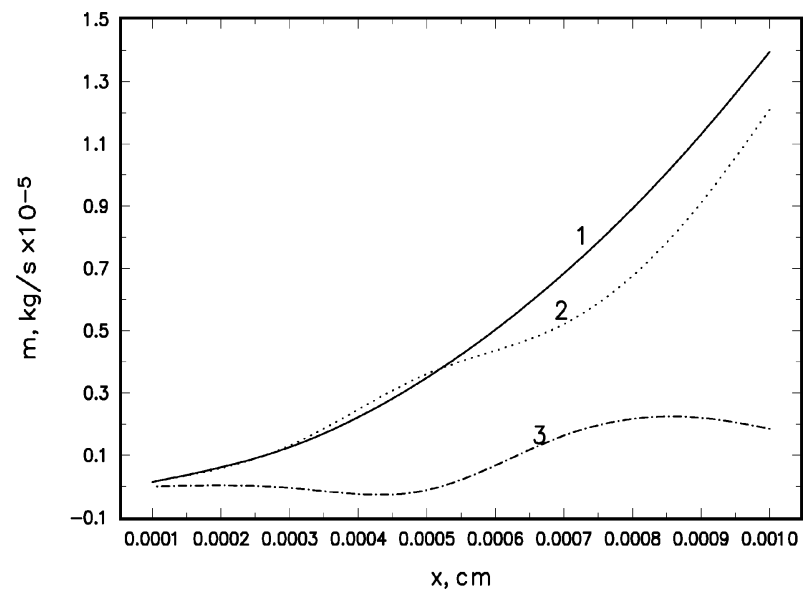

Figure 3. Mass flow rates in microwedge layer for methanolwater: (1) total contribution, (2) interfacial tension gradient contribution, (3) capillary force gradient contribution.

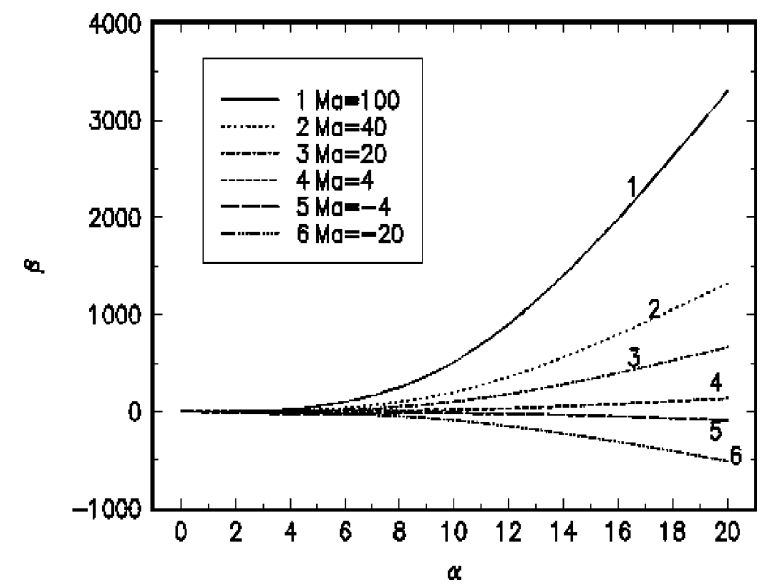

Figure 4. The variations of wave growth rate $\beta$ with wave number $\alpha$.

Expression of the wave growth rate was obtained with taking the surface gradient caused by temperature gradient and concentration gradient into account [7]. The effect of surface gradient was investigated by introducing a dimensionless number $M a$. The variation of wave growth rate $\beta$ with wave number $\alpha$ at different cases of $M a$ number is illustrated in figure 4. Evidently, it is not beneficial for stability of liquid film for positive $M a$ (it should be noted that the definition of $M a$ is

$$
M a=\frac{(\mathrm{d} \sigma / \mathrm{d} T)\left(T_{\mathrm{s}}-T_{\mathrm{w}}\right) H}{a \mu}
$$

which is different from equation (7) in sign just for the simplicity of analysis). Meanwhile, the negative value of $M a$ has the opposite effect. $M a$ is negative for evaporating 
positive binary mixtures layer and has a stabilizing effect. Therefore, the heat transfer will be significantly enhanced for evaporating positive binary mixtures. On the contrary, $M a$ value is positive for evaporating negative binary mixtures or pure liquid layer and, thus, has a destabilizing effect. Consequently, the heat transfer will be significantly deteriorated.

\subsection{Interfacial effect on heat transfer at low and moderate heat flux}

Marangoni flow convection heat transfer results from the surface tension gradient that exists at liquid-vapor interface of bubble while it is still attached to the heater surface at low and moderate heat flux. Compared with terrestrial conditions, natural convection does not exist under microgravity. However, because of continuous growth of bubble on heated surface and Marangoni force induced by large interfacial area, larger heat transfer coefficient under microgravity may be possible. By scaling analysis, Marangoni flow and associated heat transfer were studied. The Marangoni effect plays a similar role as buoyancy while the gravity is absent [8]. Nusselt number would be expressed as $N u=c M a^{1 / 3}$. Apparently, Marangoni number replaces Rayleigh number in the heat transfer correlation.

\subsection{Interfacial effect on heat transfer at high heat flux}

Macrolayer forms at high heat flux under microgravity, as usually expected [5]. Near the heater surface, vaporization is occurring more rapidly, which results in a higher local concentration of the less volatile component. Therefore, the concentration of the more volatile component will vary along the liquid-vapor interface. A gradient of concentration along the interface is established. Meanwhile, a gradient of temperature along the interface is also established along the surface because of heating. The concentration effect is often much larger than the temperature effect on surface tension. Consequently, for so-called positive binary mixtures, surface tension gradients that result in downward shear exist on the liquid-vapor interface. It is the surface tension gradients that drive liquid back to the heater surface and maintain the evaporation of macrolayer [8, 9]. Marangoni effect caused by concentration gradient plays an important role and will greatly reduce the possibility of bubble coalescence, thus, significantly enhances the value of $\mathrm{CHF}$ of positive binary mixtures. Therefore, it is easy to understand why the CHF values of positive binary mixtures under microgravity are much larger that those of negative binary mixtures and single component under microgravity $[8,9]$.

\section{EXPERIMENTAL INVESTIGATIONS}

\subsection{Experimental apparatus}

Pool nucleate boiling experiments on metal plate walls with different material and thickness were conducted to investigate the effect of interfacial behavior on nucleation boiling heat transfer of binary mixtures. The schematic of the experimental set-up is shown in figure 5(a), which mainly consists of test section, liquid tank, cooling loop, power supply and measurement system.

The working liquid was contained in a vessel, $120 \mathrm{~mm}$ in height and $110 \mathrm{~mm}$ in diameter, whose bottom was an insulation layer with two symmetrically distributed $20 \times 60 \mathrm{~mm}^{2}$ channels. Metal plate with the same size was placed into the channels as boiling wall and sealed with 704-silicone rubber. Eight copper-constantan thermocouples were embedded into the bottom of the metal plate to measure the boiling surface temperature. The film heaters were coated onto the bottom side of the metal plate whose electric resistor was $9.00 \mathrm{Ohm}$. The test section is shown in figure 5(b).

A GD1721 direct current power supply was connected in series with a standard resistor and film heater. The resistor, whose value was $2 \mathrm{Ohm}$, was used to measure the system electric current. This power supply can provide desired heat flux by adjusting the voltage input.

The date acquisition system includes one 16-channel input module (with 14 for thermocouple and 2 for voltage and electric current) whose error is $0.1 \%$ provided by the manufacturers, and an RS232 interface connected to a personal computer.

A water-cooled coil was used to minimize the loss of the test liquid and to stabilize the system pressure. Atmospheric pressure was maintained by venting the test vessel to ambient. The U-type pipe was used to monitor the pressure in the vessel. The whole test section was placed in the insulation housing to minimize the loss of heat.

\subsection{Experimental procedure and data reduction}

The parameters required to measure in these experiments are the boiling wall temperature, the voltage 


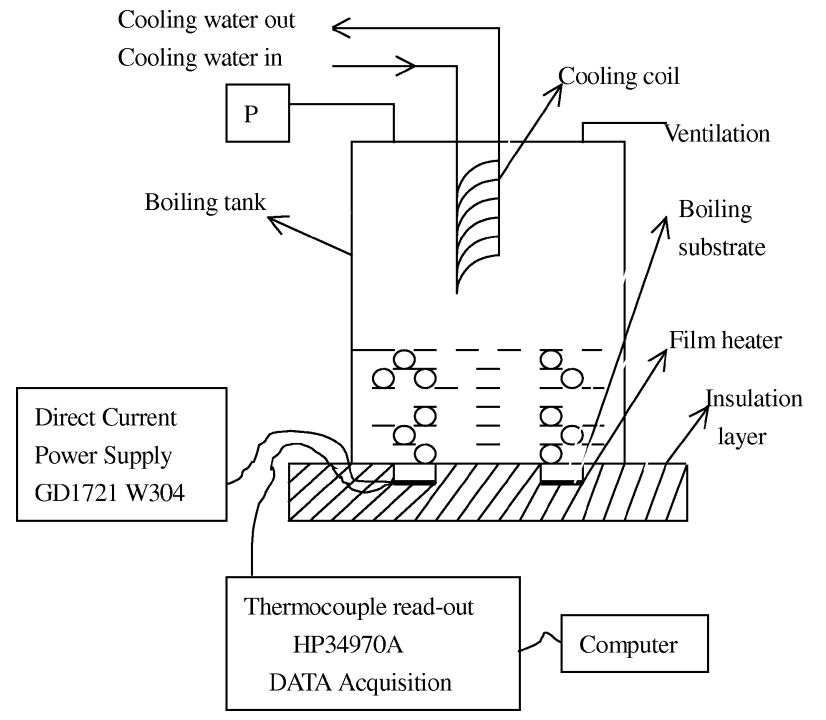

(a)
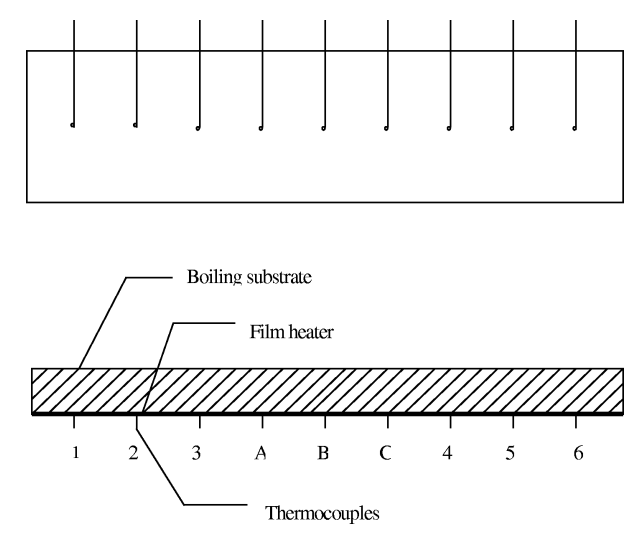

(b)

Figure 5. Experimental apparatus: (a) schematic of test apparatus, (b) boiling surface.

drop $U$ across the film heater and the current $I$. The heat flux can be calculated by the measured voltage drop $U$ across the film heater and the current $I$ as

$$
q=\frac{U I}{A}
$$

The bottom time-area averaged superheat $\Delta T$ can be obtained by data acquisition system, and then the boiling wall surface time-area averaged superheat $\Delta T_{\mathrm{w}}$ can be correlated by one-dimensional heat conduction model at each applied heat flux as

$$
\Delta T_{\mathrm{w}}=\Delta T-\frac{q b}{\lambda}
$$

Experimental procedure is as follows. First the experimental system was started up with no power input. Then it was necessary to heat the test liquid at small power until it reached the saturation temperature. Increasing the voltage input of DC power the desired heat flux was obtained. A comparison was then made between two average temperatures for each thermocouple after each heat flux increment. The procedure was repeated until the temperature difference for the thermocouples was less than $0.2 \mathrm{~K}$. The test section at this point was assumed to be at steady conditions. Usually it took about $2 \mathrm{~min}$ to reach the steady conditions after each small heat flux increment. After reaching steady state, the temperature was measured and heat flux was calculated.

Substrate heat losses were estimated to be less than $5-7 \%$ of total heat input. The uncertainty estimates in temperature were about $8 \%$. Repeated experiments showed that the data reproducibility was satisfactory.

\subsection{Results and discussions}

Boiling experiments of binary mixtures for methanolwater, methanol-n-pentane, ethanol-n-pentane and methanol-ethanol at different compositions are conducted. The heat transfer coefficient can be estimated with the measured heat flux and time-area averaged superheat as

$$
h=\frac{q}{\Delta T_{\mathrm{w}}}
$$

Figure 6 represents the experimental data for methanol-water, methanol-n-pentane, ethanol-n-pentane and methanol-ethanol mixtures. It is shown that great differences exist among these binary mixtures, which implies that the interfacial characteristics significantly affect the nucleate boiling heat transfer. The detailed analyses are as follows.

Numerous heat transfer correlations for the binary mixtures boiling heat transfer coefficient were proposed on the basis of deterioration observed in heat transfer induced by the mass transfer resistance. The most popular empirical correlation is that of Stephan and Korner [10], who suggested that wall superheat for binary mixtures boiling could be determined by the sum of ideal wall superheat $\Delta T_{\mathrm{wi}}$ and excess wall superheat $\Delta T_{\mathrm{we}}$. Ideal superheat $\Delta T_{\mathrm{wi}}$ is the superheat required when the two pure components exhibit no interactions, which is molar averaged value of the pure liquid superheats

$$
\Delta T_{\mathrm{wi}}=x_{1} \Delta T_{\mathrm{w} 1}+\left(1-x_{1}\right) \Delta T_{\mathrm{w} 2}
$$




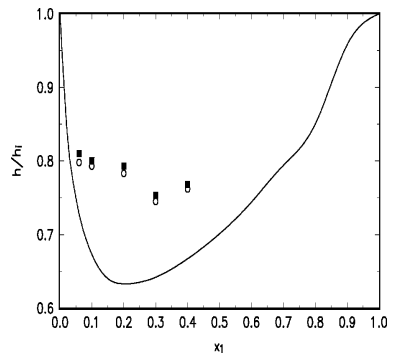

(a)

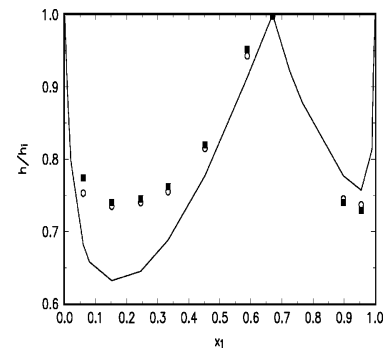

(c)

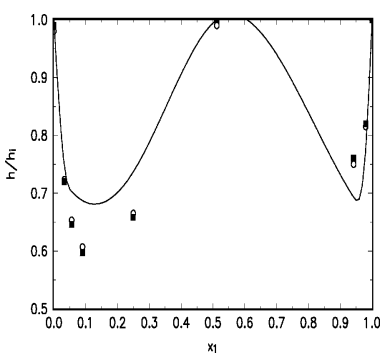

(b)

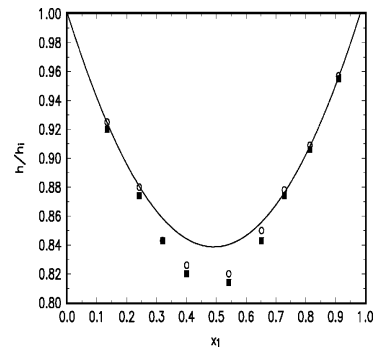

(d)
Figure 6. Comparisons of experimental data with predictions: (a) water-methanol; solid points, $q=34.75 \mathrm{~kW} \cdot \mathrm{m}^{-2}$; empty points, $q=17.82 \mathrm{~kW} \cdot \mathrm{m}^{-2}$; line, equation (15); (b) n-pentane-methanol; solid points, $q=32.82 \mathrm{~kW} \cdot \mathrm{m}^{-2}$; empty points, $q=19.11 \mathrm{~kW} \cdot \mathrm{m}^{-2}$; line, equation (15); (c) n-pentane-ethanol; solid points, $q=31.11 \mathrm{~kW} \cdot \mathrm{m}^{-2}$; empty points, $q=16.57 \mathrm{~kW} \cdot \mathrm{m}^{2}$; line, equation (15); (d) ethanolmethanol; solid points, $q=32.82 \mathrm{~kW} \cdot \mathrm{m}^{-2}$; empty points, $q=17.82 \mathrm{~kW} \cdot \mathrm{m}^{-2}$; line, equation (15).

where $\Delta T_{\mathrm{w} 1}$ and $\Delta T_{\mathrm{w} 2}$ are the wall superheats required for boiling of pure component 1 and component 2 at same wall and heat flux, which can be calculated by pool nucleate boiling heat transfer correlation of single liquid [11]

$$
\frac{c_{\mathrm{pl}} \Delta T}{L}=c_{\mathrm{sf}}\left\{\frac{q}{\mu_{1} L}\left[\frac{\sigma}{g\left(\rho_{1}-\rho_{\mathrm{v}}\right.}\right]^{1 / 2}\right\}^{0.33} \operatorname{Pr}^{1.7}
$$

So, ideal boiling heat transfer of binary mixtures is

$$
h_{\mathrm{i}}=\frac{q}{\Delta T_{\mathrm{wi}}}
$$

The excess superheat is calculated as

$$
\Delta T_{\mathrm{we}}=A_{0}(0.88+1.2 P)(y-x) \Delta T_{\mathrm{wi}}
$$

for present mixtures, where $A_{0}=1.53$. The ratio for the actual heat transfer coefficient and the ideal heat transfer coefficient is [10]

$$
\frac{h}{h_{\mathrm{i}}}=\frac{1}{1+A_{0}(0.88+1.2 P)(y-x)}
$$

The curves in figure 6 illustrate the results calculated by equation (15).

As shown in figure 6, the deviations made between the theoretical results and experimental data are apparent, which can be attributed to the interfacial effects. For positive binary mixtures, the experimental data are larger than theoretical results, while for negative binary mixtures, the experimental data are smaller than theoretical results. In addition, the larger the absolute values of $M a$, the more evident the deviation between the theoretical results and experimental data will be. The deviation is more evident at high heat flux than at low heat flux. In a word, the deterioration and enhancement for binary mixtures boiling can both be attributed to the interfacial Marangoni effect.

It is necessary to include the Marangoni effect into the available model to accurately calculate the nucleate boiling heat transfer of binary mixtures. An empirical correlation, with the dimensionless number $M a$ defined by equation (7), fitting the present experimental data, is given as [1]

$$
\frac{h}{h_{\mathrm{i}}}=\frac{1}{1+A_{0}(0.88+1.2 P)(y-x)}\left(1+m \frac{|M a|^{n}}{M a}\right)
$$

For low heat flux, $m=1.5 \cdot 10^{-3}, n=1.39$; for high heat flux, $m=1.5 \cdot 10^{-3}, n=1.45$. At high heat flux, the mechanism of interfacial effects is similar to that of CHF [12], the present modified correlation may also be extended to calculate the value of CHF.

\section{CONCLUSIONS}

Effects of interfacial behavior on nucleate boiling bubble dynamics and associated heat transfer of binary mixtures for ethanol-water, methanol-water, methanoln-pentane, ethanol-n-pentane and methanol-ethanol are theoretically and experimentally analyzed. Marangoni effect plays an important role in nucleate boiling heat transfer of binary mixtures besides mass transfer resistance. The theoretical results would not correlate with experimental results if the Marangoni effects at interface had not been considered.

\section{Acknowledgement}

The project was supported by the National Natural Science Foundation of China through contracts \#59625612 and \#59976016. 
L.H. Chai et al.

\section{REFERENCES}

[1] Fujita Y., Bai Q., Critical heat flux of binary mixtures in pool boiling and its correlation in terms of Marangoni number, Int. J. Refrig. 20 (1997) 616-622.

[2] Celata G.P., Cumo M., Setaro T., A review of pool and forced convection boiling of binary mixtures, Exp. Thermal Fluid Sci. 9 (1994) 367-387.

[3] Jasper J.J., The surface tension of pure liquid compounds, J. Phys. Chem. Ref. Data 1 (1972) 841-1010.

[4] Edwards D.K., Heat Exchanger Design Handbook, Vol. 5, Physical Properties, Hemisphere, New York, 1983.

[5] Chai L.H., Binary mixtures boiling under microgravity and nonlinear features of boiling, Ph.D. Thesis, Tsinghua University, China, 1999.

[6] Chai L.H., Peng X.F., Wang B.X., Ochterbeck J.M., Interfacial behavior of growing bubbles in concentration boundary layer, Int. J. Heat Mass Tran. 41 (1998) 35293535.
[7] Chai L.H., Peng X.F., Ochterbeck J.M., Stability analysis of an evaporating and/or condensing thin liquid film, in: Proceedings of the 1998 ASME International Mechanical Engineering Congress and Exposition, Anaheim, CA, USA, 1998, pp. 325-329.

[8] Chai L.H., Peng X.F., Ochterbeck J.M., Nucleate pool boiling heat transfer of binary mixtures under microgravity, in: AIAA 1997 National Heat Transfer Conference, Baltimore, MD, USA, 1997.

[9] Chai L.H., Peng X.F., Nucleate boiling of binary mixture at high heat flux under microgravity, Journal of Aerospace Power 13 (1998) 263-266 (in Chinese).

[10] Stephan K., Korner M., Calculation of heat transfer in evaporating binary liquid mixtures, Chem. Ing. Tech. 41 (1969) 409-417.

[11] Rohsenow W.M., A method of correlating heat transfer data for surface boiling liquids, J. Heat Tran. 74 (1952) 969.

[12] McGillis W.R., Carey V.P., On the role of Marangoni effects on the critical heat flux for pool boiling of binary mixtures, J. Heat Tran. 118 (1996) 103-109. 